\title{
On the importance of $\pi-\pi$ stacking and cation-anion interactions in the construction of non-centrosymmetric networks of bromide salts of imidazolium cations bearing arene and polyfluoroarene rings
}

\author{
G. C. Saunders \\ CrystEngComm, 2011, 13, 1801-1803
}

Requests for reprints should be made to g.saunders@waikato.ac.nz

Abstract: The salt 1-(2,3,5,6-tetrafluoropyridyl)-3-benzylimidazolium bromide crystallizes in the non-centrosymmetric space group $P$ na $2{ }_{1}$. The structure arises from $\pi-\pi$ stacking between the benzyl and tetrafluoropyridyl groups of the cations and cation-bromide interactions. It is the latter that gives rise to the non-centrosymmetry.

The ' $\pi-\pi$ stacking' interaction between complementary arenes and polyfluoroarenes has received some attention as a tool in crystal engineering. ${ }^{1,2}$ Typically studies have concentrated on molecules in which complementary rings of a molecule can be co-planar, and which typically pack such that the arene and polyfluoroarene of one molecule stack with the complementary rings of two other molecules forming columns that are virtually perpendicular to the rings (Scheme 1a). The alternating direction of the polarity of the molecules arising from this stacking results in no net polarity. Consequently, in the absence of other factors, these molecules don't crystallize in a non-centrosymmetric crystal class.

Recently the design and construction of a non-centrosymmetric network incorporating $\pi-\pi$ stacking interactions between benzyl and pentafluorobenzyl groups was reported. ${ }^{3}$ It was argued that facilitating the bifurcated stacking of the rings, by preventing columnar stacking, would generate a polar axis necessary for non-centrosymmetry (Scheme 1b). It was reasoned that columnar stacking could be prevented by the use of molecules in which the planes of the complementary rings can be parallel but not co-planar, and in which the 'step' is greater than $4 \AA$. (This would also prevent weaker stacking interactions between the imidazolium rings. ${ }^{4}$ ) The cation of 1-(2,3,4,5,6-pentafluorobenzyl)-3-benzyl-imidazolium bromide, 1, satisfies the criteria and was indeed found to crystallize in the non-centrosymmetric space group $P 1$ (BONKUL). ${ }^{3}$ The $\pi-\pi$ stacking of cations of 1 generates chains of cations parallel to the $c$ axis with columns of complementary rings stacked parallel to the $b$ axis, forming sheets (Scheme 
1c). However the $\pi-\pi$ stacking has no obvious influence on the structure parallel to the $a$ axis, and a centrosymmetric structure would result if alternating sheets were arranged antiparallel. It is therefore likely that the interactions between the cations and the bromide anions are more important in determining the symmetry of the crystal structure of $\mathbf{1}$ than the $\pi-\pi$ stacking interactions. The cation-bromide interactions hold the sheets of cations parallel and additionally orientate the imidazole rings in the same direction $\left(\mathrm{N}_{2} \mathrm{C}-\mathrm{H}\right.$ lies almost parallel to the $a$ axis). Each bromide bridges two sheets with close contacts to the acidic hydrogen atom of the imidazolium ring $(\mathrm{C} \cdots \mathrm{Br} 3.678(6) \AA)$ of one cation and a backbone hydrogen atom of an imidazolium ring of a neighbouring sheet $(\mathrm{C} \cdots \mathrm{Br} 3.619(6) \AA)$. These data are suggestive of hydrogen bonding, ${ }^{5}$ as has been described in other imidazolium bromides. ${ }^{4}$ The importance of the bromide anion in the crystal structure is supported by the lack of crystallization of the chloride, iodide and perchlorate salts. ${ }^{3}$

$\pi-\pi$ stacking can also occur between complementary rings of angular molecules. Although in the crystal structure of the tick- or check mark-shaped molecule 4$\mathrm{C}_{6} \mathrm{H}_{5} \mathrm{OC}_{6} \mathrm{~F}_{4} \mathrm{C} \equiv \mathrm{CC}_{6} \mathrm{H}_{5}$ the fluoroarene stacks preferentially with the phenyl ring with which it is coplanar, ${ }^{2}$ columnar $\pi-\pi$ stacking is present in the crystal structure of the chevron-shaped molecule 1,2,3,4-tetrafluorodibenzo[b,f][1,4]-oxazepine (HUTYAW), ${ }^{6}$ in which the planes of the phenylene and tetrafluorophenylene rings subtend an angle of $c a .144^{\circ}$ at the hinge. The relatively obtuse angle at the hinge and the equidistance of the rings from the hinge allows the sufficiently close approach of the molecules to give rise to stacking. (The distances between the planes of complementary rings are $c a .3 .3$ and $3.45 \AA$ A.) Consequently the crystal structure contains parallel columns of alternating enantiomers (Scheme 2a). The direction of the bend generates polarity along the column, but this alternates between the columns giving rise to centrosymmetry $\left(P 2_{1} / \mathrm{n}\right)$. It is expected that columnar stacking between angular building blocks can be prevented by using complementary rings non-equidistant from the hinge, so allowing bifurcated stacking (Scheme 2b).

The salt 1-(2,3,5,6-tetrafluoropyridyl)-3-benzylimidazolium bromide, $\mathbf{2}$, is similar to $\mathbf{1}$, but lacks one methylene group and consequently the cation is tick-shaped (the complementary rings are not equidistant from the hinge) and the planes of the complementary rings cannot be parallel. Consequently bifurcated stacking is expected. In addition the likelihood of imidazolium $\cdots$ bromide interactions similar to those of $\mathbf{1}$ suggest that the crystal structure of $\mathbf{2}$ would be useful in understanding the factors important in determining the symmetry of crystal structures of salts of imidazolium cations bearing arene and polyfluoroarene rings, especially 
the relative importance of $\pi-\pi$ stacking and cation-anion interactions.

Salt 2, the synthesis of which will be reported elsewhere, crystallized in the noncentrosymmetric space group Pna2 ${ }_{1}$ in the orthorhombic crystal system. ${ }^{\dagger}$ The benzylimidazole moiety (Figure 1) is similar to that of $\mathbf{1}^{3}$ The hinge angle, $\left((\mathrm{Ph}) \mathrm{C}-\mathrm{CH}_{2}-\mathrm{N}\right), 110.68(15)^{\mathrm{o}}$, and the $\mathrm{C}-\mathrm{N}-\mathrm{CH}_{2}-\mathrm{C}$ torsion angles, $98.1(1)^{\circ}$ and $-80.2(2)^{\circ}$, are similar to those in $\mathbf{1}$, although the $\mathrm{C}-\mathrm{C}-\mathrm{CH}_{2}-\mathrm{N}$ torsion angles differ significantly: $81.7(2)^{\circ}$ and $-96.1(2)^{\circ}$ for $\mathbf{2}$, $73.82^{\circ}$ and -107.52 for 1 . The plane of tetrafluoropyridyl ring is twisted from co-planarity with the imidazolium ring by $c a .39 .5^{\circ}$.

The phenyl and the tetrafluoropyridyl ring centroids are 2.90(3) and 6.25(3) $\AA$ respectively from the hinge (C9) and consequently columnar stacking is prevented and bifurcated $\pi-\pi$ stacking is observed. Interacting rings are virtually parallel, deviating by only ca. $4.8^{\circ}$, and are offset by ca. $1.2 \AA$ (Figure 2). The inter-ring separation ranges from 3.26(3) $\AA\left(\mathrm{N} 3 \cdots \mathrm{C}_{6}\right)$ to $3.38(5) \AA\left(\mathrm{C} 14 \cdots \mathrm{C}_{5} \mathrm{~F}_{4} \mathrm{~N}\right)$. This contrasts with the structure of $\mathbf{1}$ in which the offset is $c a$. $0.4 \AA$ smaller, but the inter-ring separation is $c a$. $0.2 \AA$ greater. The fluoroarene ring also shows an interaction with a bromide anion $\left(\mathrm{Br} \cdots \mathrm{C}_{5} \mathrm{~F}_{4} \mathrm{~N}\right.$ 3.32(3) $\AA$, Br ‥C5 3.398(3) $\AA, \quad \mathrm{Br} \cdots \mathrm{C} 43.441(3) \AA$ ), such that the $\mathrm{C} 14-\mathrm{C}_{5} \mathrm{~F}_{4} \mathrm{~N}_{\text {centroid }}-\mathrm{Br}$ angle is almost linear $\left(171.8(3)^{\circ}\right)$. Stacking between cations gives infinite chains of alternating enantiomers parallel to the $a$ axis (Figure 3 ). The chains are identical parallel to the $b$ axis, but alternate in polarity parallel to the $c$ axis. Consequently there is no nett polarity parallel to the $a$ and $b$ axes. However, non-centrosymmetry is generated by the imidazole rings aligning in the same direction giving a nett polarity parallel to the $c$ axis.

As with the structure of $\mathbf{1}$, it is evident that the bromide anion plays a pivotal role in determining the crystal structure. ${ }^{3}$ The arrangement around the bromide is close to trigonal planar: $\mathrm{C} 1 \cdots \mathrm{Br} \cdots$ pyF $116.0(2)^{\mathrm{o}}, \mathrm{C} 3 \cdots \mathrm{Br} \cdots$ pyF $122.7(2)^{\mathrm{o}}, \mathrm{C} 1 \cdots \mathrm{Br} \cdots \mathrm{C} 3121.1(2)^{\mathrm{o}}$ (pyF represents the centroid of the tetrafluoropyridyl ring). As well as an interaction with the fluoroarene, each bromide ion is close to the hydrogen atoms of imidazole rings (Figure 4). The geometry about $\mathrm{C} 1$ and the $\mathrm{C} 1 \cdots \mathrm{Br}$ distance (3.467(3) $\AA$. $c f$. $\Sigma$ van der Waals radii $3.55^{7}$ ) suggest hydrogen bonding between the acidic hydrogen, H1, and the bromide anion. ${ }^{5}$ The

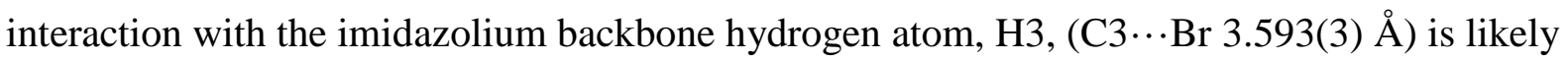
to be weaker.

Attempts to prepare the tetrafluoroborate salt of $\mathbf{2}$ by anion metathesis in methanol using a large excess of sodium tetrafluoroborate were unsuccessful. This observation suggests a larger lattice enthalpy for the bromide salt than the tetrafluoroborate salt and further 
demonstrates the importance of bromide in the crystal structure.

The data for $\mathbf{1}$ and $\mathbf{2}$ strongly suggest that $\pi-\pi$ stacking is important for the formation of chains and sheets with polarity in one or two directions, but cannot account for polarity in the perpendicular direction. In salts $\mathbf{1}$ and $\mathbf{2}$ this arises from hydrogen bonding between the imidazolium rings and the bromide anions.

1 For example: J. C. Collings, K. P. Roscoe, E. G. Robins, A. S. Batsnaov, L. M. Stimson, J. A. K. Howard, S. J. Clark and T. B. Marder, New J. Chem., 2002, 26, 1740; J. C. Collings, P. S. Smith, D. S. Yufit, A. S. Batsnaov, J. A. K. Howard and T. B. Marder, CrystEngComm, 2004, 6, 25; J. C. Collings, A. S. Batsnaov, J. A. K. Howard and T. B. Marder, Can. J. Chem., 2006, 84, 238.

2 C. E. Smith, P. S. Smith, R. Ll. Thomas, E. G. Robins, J. C. Collings, C. Dai, A. J. Scott, S. Borwick, A. S. Batsnaov, S. W. Watt, S. J. Clark, C. Viney, J. A. K. Howard, W. Clegg and T. B. Marder, J. Mater. Chem., 2004, 14, 413.

3 J. M. Serrano-Becerra, S. Hernández-Ortega, D. Morales-Morales and J. Valdés-Martínez, CrystEngComm, 2009, 11, 226.

4 L. Leclercq, N. Boujeim, A. R. Schmitzer, Cryst. Growth Des., 2009, 9, 4784.

5 G. R. Desiraju, Angew. Chem. Int. Ed., 2011, 50, 52.

6 C. L. Allaway, M. Daly, M. Nieuwenhuyzen and G. C. Saunders, J. Fluorine Chem., 2002, 115,91 .

7 A. Bondi, J. Phys. Chem., 1964, 68, 441; R. S. Rowland and R. Taylor, J. Phys. Chem., 1996, 100, 7384.

$\dagger$ AMOCOV; CCDC 806231. Crystallographic data for 2 (gold prism): $\mathrm{C}_{15} \mathrm{H}_{10} \mathrm{~F}_{4} \mathrm{~N}_{3}, \mathrm{Br}, \mathrm{M}$ 388.17, orthorhombic, $P$ na2 $1, a=12.7109(3), b=9.5071(3), c=12.7444(4) \AA, V=1540.08(8)$ $\AA^{3}, Z=4, D_{\text {calc }}=1.674 \mathrm{gcm}^{-3}, \mu($ Mo K $\alpha) 2.712 \mathrm{~mm}^{-1}$, crystal size $0.67 \times 0.60 \times 0.30 \mathrm{~mm}$. Data were collected at $90 \mathrm{~K}$ on a Bruker APEX II CCD area detector using Mo Ka radiation. A total of 36035 reflections $\left(2.67<\theta<29.05^{\circ}\right)$ were collected, of which 3706 were unique with $I>$ $2 \sigma(I)$. The structure was solved by direct methods using SHELXS-97 and refined using 
SHELXL-97 with all non-H atoms anisotropic. $R_{\text {int }}=0.0367, R_{1}=0.0214, w R_{2}=0.0528$, GOF $=1.047$, Flack parameter $=0.008(6)$.

Figure 1:

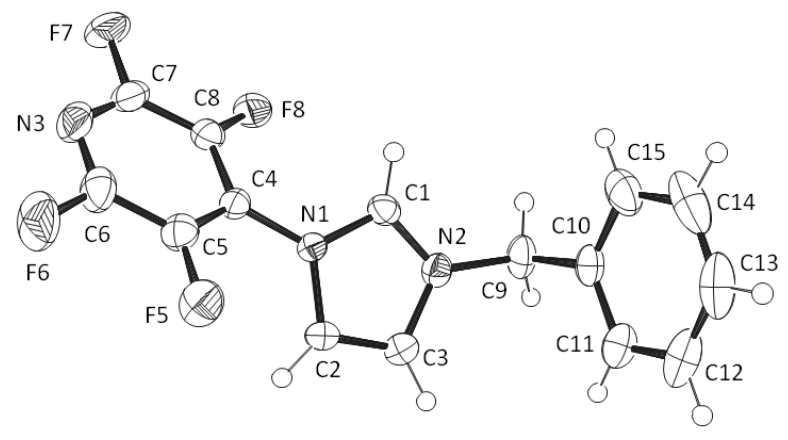

Figure 2:

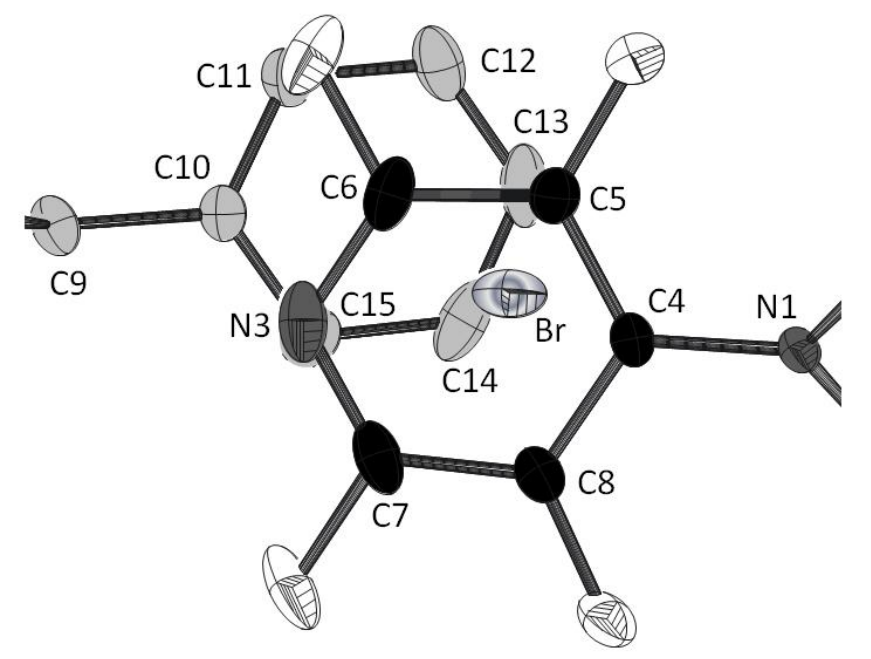


Figure 3:

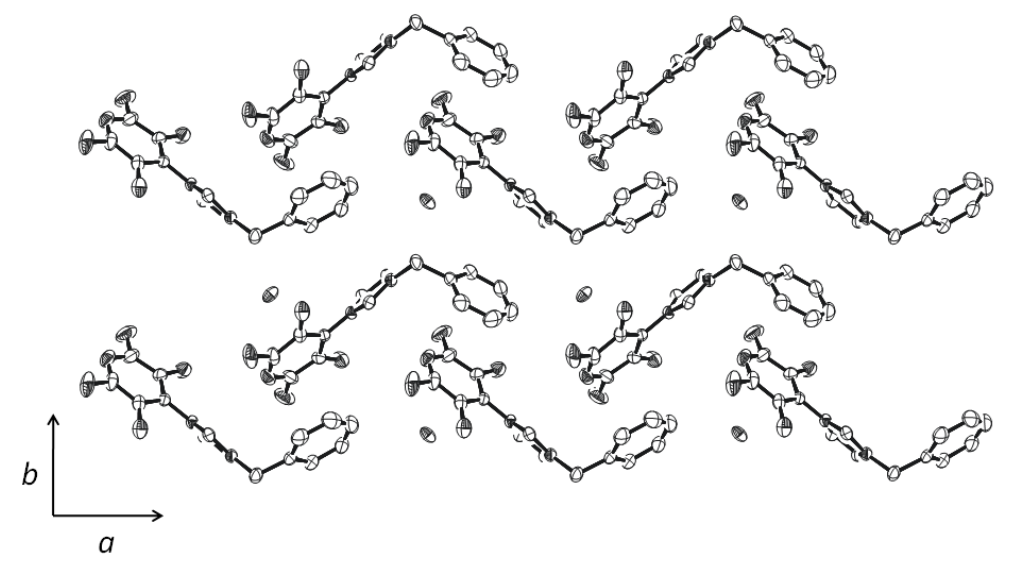

Figure 4:

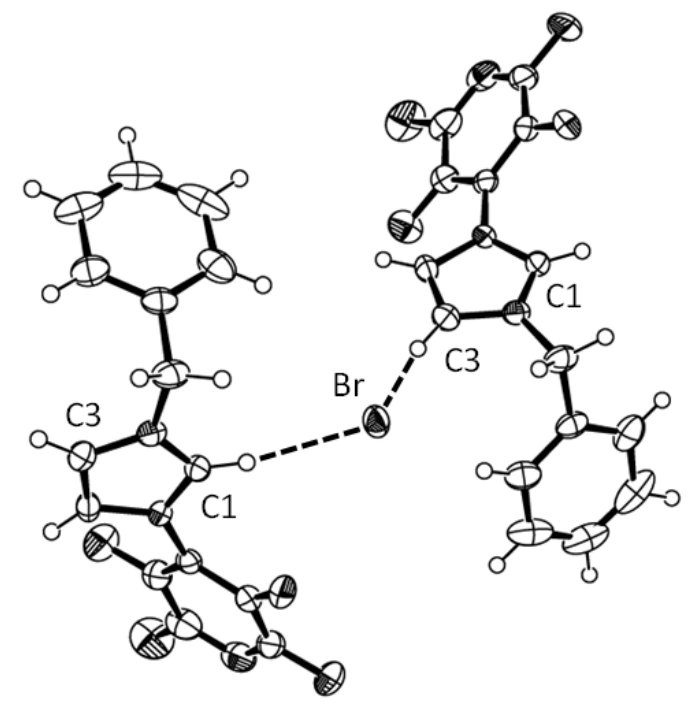


Scheme 1
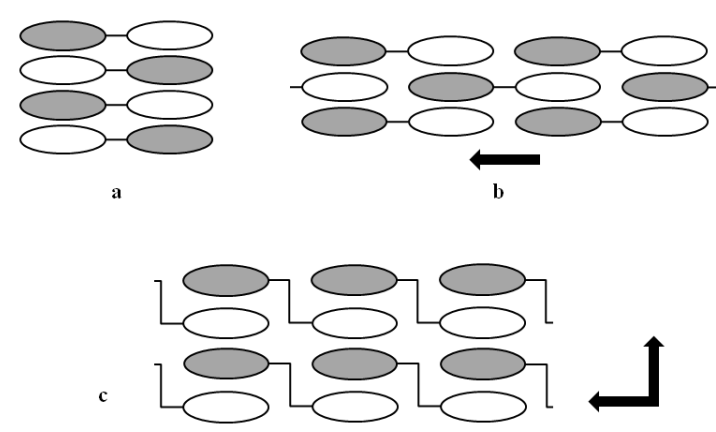

Scheme 2

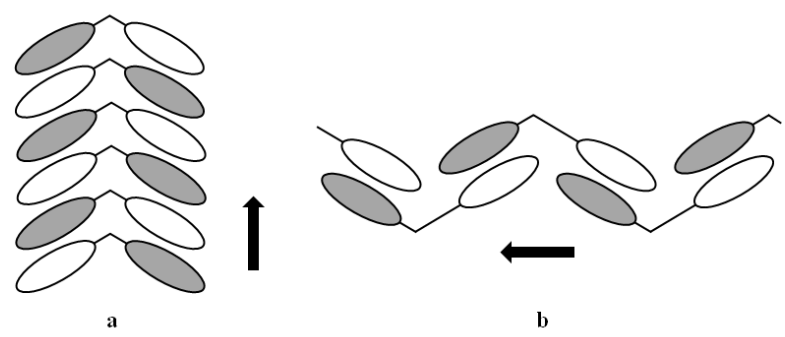

